\title{
PAPER \\ Isosceles-Trapezoidal-Distribution Edge Tapered Array Antenna with Unequal Element Spacing for Solar Power Satellite
}

\author{
A.K.M. BAKI ${ }^{\dagger * a)}$, Kozo HASHIMOTO ${ }^{\dagger}$, Naoki SHINOHARA ${ }^{\dagger}$, Tomohiko MITANI ${ }^{\dagger}$, Members , \\ and Hiroshi MATSUMOTO ${ }^{\dagger}$, Fellow
}

\begin{abstract}
SUMMARY The Earth will require sustainable electricity sources equivalent to 3 to 5 times the commercial power presently produced by 2050. Solar Power Satellite (SPS) is one option for meeting the huge future energy demand. SPS can send enormous amounts of power to the Earth as the form of microwave (MW). A highly efficient microwave power transmission (MPT) system is needed for SPS. A critical goal of SPS is to maintain highest Beam Efficiency (BE) because the microwaves from SPS will be converted to utility power unlike the MW from communication satellites. Another critical goal of SPS is to maintain Side Lobe Levels (SLL) as small as possible to reduce interference to other communication systems. One way to decrease SLL and increase BE is the edge tapering of a phased array antenna. However, tapering the excitation requires a technically complicated system. Another way of achieving minimum SLL is with randomly spaced element position but it does not guarantee higher BE and the determination of random element position is also a difficult task. Isosceles Trapezoidal Distribution (ITD) edge tapered antenna was studied for SPS as an optimization between full edge tapering and uniform amplitude distribution. The highest Beam Collection Efficiency (BCE) and lowest SLL (except maximum SLL) are possible to achieve in ITD edge tapering and ITD edge tapered antenna is technically better. The performance of ITD is further improved from the perspective of both Maximum Side Lobe Level (MSLL) and BE by using unequal spacing of the antenna elements. A remarkable reduction in MSLL is achieved with ITD edge tapering with Unequal element spacing (ITDU). BE was also highest in ITDU. Determination of unequal element position for ITDU is very easy. ITDU is a newer concept that is experimented for the first time. The merits of ITDU over ITD and Gaussian edge tapering are discussed.

key words: SPS, microwave power transmission, phased array, unequally spaced array, antenna tapering
\end{abstract}

\section{Introduction}

The demand for SPS in the 21st Century is discussed in details in [1]. The prosperity of human being on Earth by 2050 will require sustainable electricity sources equivalent to 3 to 5 times the commercial power presently produced [2]. Global warming is another key problem in the 21st century. Electricity generated from any renewable energy source, such as hydro, wind, biomass, geothermal and solar, is considered "green" because of the negligible impact on greenhouse gas emissions [3]. One source of clean, safe, low-cost commercial electricity from solar power via the moon is described in [2]. The first idea of Solar Power Satellite (SPS) was conceived by Peter Glaser in 1968 [4]. The idea of

\footnotetext{
Manuscript received March 9, 2007.

Manuscript revised June 22, 2007.

${ }^{\dagger}$ The authors are with Research Institute for Sustainable Humanosphere (RISH), Kyoto University, Uji-shi, 611-0011 Japan.

${ }^{*}$ Presently, with the Independent University, Bangladesh.

a)E-mail: baki@secs.iub.edu.bd

DOI: 10.1093/ietcom/e91-b.2.527
}

Glaser paved the way for DOE/NASA study of microwave (MW) power transmission from satellite. Solar panels of the SPS would be placed in geostationary orbit (GEO) at a distance $36000 \mathrm{~km}$ from the Earth's surface. Beam Efficiency (BE)/Beam Collection Efficiency (BCE) and Maximum Side Lobe Level (MSLL) are the indices for the evaluation of the MW beam. BE is the ratio of energy flow within the main beam to the whole transmitted power. Similarly BCE of the Microwave Power Transmission (MPT) systems is the ratio of energy flow that is intercepted by the rectenna to the whole transmitted power. The word "rectenna" is derived from the words "rectifying antenna." Rectenna is the receiving antenna for the MW power from the SPS. The received MW power on the rectenna will be converted to the utility power. BE for two dimensional beam pattern quantifies the solid angle extent of the main beam relative to that of the entire pattern and can be expressed as [5]:

$$
B E_{2 D}=\iint_{\text {main_beam }}|P(\theta, \phi)|^{2} d \Omega / \iint_{4 \pi}|P(\theta, \phi)|^{2} d \Omega
$$

Here, $P(\theta, \phi)$ is the radiated electric field.

$\mathrm{BE}$ for one dimensional case can be expressed as:

$$
B E_{1 D}=\left.\int_{\theta_{m}}|P(\theta)|^{2} d \theta\left|\int_{\theta_{w}}\right| P(\theta)\right|^{2} d \theta
$$

$\theta_{m}$ is the angle sector due to one dimensional main beam and $\theta_{w}$ is the angle sector of $\pm 90^{\circ}$.

$P(\theta)$ is the one dimensional radiated electric field.

Equations for the calculation procedure of $\mathrm{BCE}$ are described in [6] those are very similar to Eqs. (1) and (2). The numerator of the above equations is to be replaced by the size of rectenna during the calculation of BCE.

Suppression of Grating Lobes (GL) and Side Lobe Levels (SLL) is necessary for higher BCE and to avoid interference to other communication systems. If GL appear and SLL increase, the transmitted power will be absorbed into these lobes which will cause reduction of $\mathrm{BE} / \mathrm{BCE}$ and interference will also increase. A phased array antenna has been proposed in SPS system to maintain higher BCE. If all antennas are uniformly excited then the main beam will carry only a part of the total energy due to higher SLL. The targeted BCE for SPS is $90 \%$ to reduce the cost. It has been seen that it is possible to increase BCE and reduce SLL if edge tapering system can be adopted in MPT antennas.

An optimized circularly symmetrical amplitude distribution for MPT is described in [7] to achieve maximum 
transmission efficiency with constraint on the power levels outside the receiving aperture. A $10 \mathrm{~dB}$ Gaussian taper has been discussed in [8]-[10] and has been proposed in the NASA/DOE model [11] and JAXA model. Other methods of maximizing the electromagnetic power with some constraints have been discussed in [12], [13]. A conceptual study of amplitude tapering effect for low SLL has been presented in [14]. Taylor aperture distribution pattern has been presented in [15] for array design. A new approach of designing Chebyshev array has been presented in [16] where some new equations have been derived to estimate the excitation currents. Though the best form of amplitude distribution is known to be the Gaussian at the same time it has the demerits of poor use of radiating antenna surface [17] and the references there in]. One possible solution is described in [18] by introducing discontinuous antenna radiators distribution. A comparison between Taylor amplitude tapered array and statistically thinned array is shown in [19]. The main beam and the near in SLL behavior of the two patterns were similar but the SLL of the statistically designed thinned array was higher than that of the Taylor design over a large part of the angular region [Fig. 3 of 19]. Though this difference is not that important for many other practical array applications but this will cause a reduction of BCE in MPT application. Therefore MPT designers must be concerned with the BE/BCE when they design phased array with thinned array, or random array concepts. Moreover the manufacturability of thinned/random array needs to be accounted for during design cycle. It is mentioned in [20] according to the reference [21] that almost $100 \%$ efficiency can be achieved by edge tapering which is essentially Gaussian distribution [Fig. 2, Fig. 3 of reference 20 and Fig. 5 of reference 21]. Optimized Gaussian tapering can be used in MPT to increase the energy transmission efficiency. But the effectiveness of MPT also depends on the uniformity of amplitude distribution along the surface, i.e. maximum utilization of antenna surface. It has been discussed in [6] that edge tapering for SPS like optimized Gaussian, Chebyshev, and Taylor distribution has some complexity of different power output levels at different antennas and problem of heat radiation and an optimization was proposed between optimized Gaussian distribution and uniform amplitude distribution. It was shown [6] that the highest BCE and lowest SLL (except MSLL with lower number of antennas to be tapered) can be achieved with ITD. ITD edge tapering is comparatively a new idea of phased array power distribution based on Isosceles Trapezoid. A most technological and effective method is needed for MPT. The amplitude distribution in ITD is almost uniform which is technically easy to build and better use of antenna surface can be achieved by ITD. Even in optimized Gaussian distribution the use of antenna surface is still poor. The performance of ITD is further improved from the perspective of both Maximum Side Lobe Level (MSLL) and $\mathrm{BE} / \mathrm{BCE}$ by using combined equal and unequal spacing of the antenna elements.

The amplitude distribution of ITDU is same as that of ITD and is also technically better than that of Gaussian dis- tribution. The unequal element spacing is derived from the ITD concept and by using the sinc function. No grating lobe in the observation range of $\pm 90^{\circ}$ is formed due to unequal spacing with ITD. The determination of unequal element spacing for ITDU is much easier than that of thinned array or random array and it will be technically easier to be built.

An experimental result on ITDU is discussed. The mutual coupling effect is neglected in this paper. The validity of ITDU by using element spacing range of $0.6 \lambda-0.8 \lambda$ and beam steering angle of $10^{\circ}$ with the array broadside is discussed. For real SPS application the beam steering angle will be within $\pm 5^{\circ}$ with the array broadside and it is possible to use the antenna spacing between $0.58-0.8 \lambda$ for SPS system and it would be possible to push the mutual coupling to lower levels. The authors' group is presently working on the effect of mutual coupling. It is negligible under such spacings. The performance of ITDU for two dimensional (2D) arrays is also discussed.

\section{Isosceles Trapezoidal Distribution Tapering}

The concept of ITD for one dimensional (1D) array is shown in Fig. 1. In this method amplitude of only a few edge transmitting antennas/units are tapered. Amplitudes of remaining most antennas/units are uniform. In recent SPS design, a concept of "unit" is adopted. Each unit consists of several phased array antenna elements. For example 25 elements or 10 elements can be considered as a "unit."

Array Factor (AF) with ITD is:

$$
A F=\sum_{n=1}^{N_{t}} A_{t n} e^{j n \psi_{1}}+\sum_{m=1}^{N} e^{j\left(m+N_{t}\right) \psi}+\sum_{n=1}^{N_{t}} A_{t n} e^{j\left(n+N_{t}+N\right) \psi_{2}}
$$

where, $N=$ Number of SPS units with uniform amplitudes,

$N_{t}=$ Number of units tapered from each side,

$$
\begin{aligned}
& \psi=\beta D_{u}\left(\sin \theta-\sin \theta_{0}\right) . \\
& \psi_{1}=-\beta D_{u}\left(\sin \theta-\sin \theta_{0}\right) . \\
& \psi_{2}=\left[(N-1) D_{u}+n D_{u}\right]\left(\sin \theta-\sin \theta_{0}\right) \beta .
\end{aligned}
$$

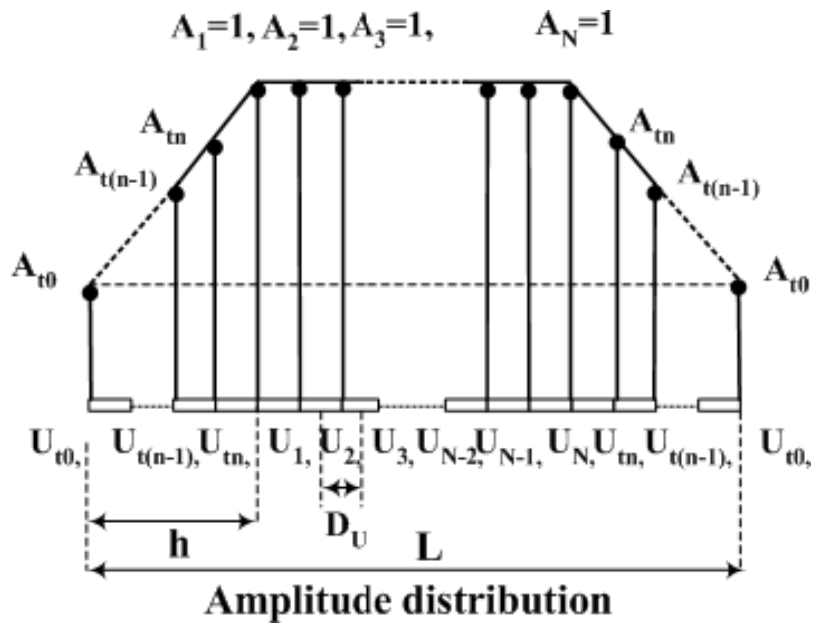

Fig. 1 ITD type edge tapering for SPS transmitting antennas. 
$D_{u}=$ inter-unit spacing $(\mathrm{m})$.

$N_{T}=$ Total number of antenna units $=N+2 N_{t}$.

$\beta=2 \pi / \lambda=$ phase constant.

The amplitude distribution of the ITD tapering is:

$$
A_{t n}=\left(1-A_{t 0}\right) n / N_{t}+A_{t 0}
$$

$A_{t 0}$ is the amplitude of each of the end units.

$\theta_{0}=$ Direction of beam maximum along the broad side.

$$
n=0,1,2 \ldots N_{t}, \quad 0 \leq A_{t n} \leq 1
$$

In Fig. 1,

$\mathrm{h}=$ Length of the antennas tapered from each side.

$\mathrm{L}=$ Total length of the array

$\mathrm{A}_{1}=\mathrm{A}_{2}=\mathrm{A}_{3}=\ldots=\mathrm{A}_{\mathrm{N}}=1$ (normalized maximum power). $m=1,2,3 \ldots N$.

\section{Determination of Unequal Element Spacing for ITDU}

The Isosceles Trapezoidal Distribution in Sect. 2 is explained for the power distribution of antenna units. The same ITD expression (Eq. (4)) can be used when antenna elements are considered instead of antenna "units" and can be used for the determination of unequal spacing of phased array antenna elements. Let us consider the isosceles trapezoidal amplitude distribution is $A(x)$ which is similar to Eq. (4). Here $A(x)$ is only the amplitude distribution of an isosceles trapezoid and not the power distribution of the antennas as is considered in Sect. 2. The variable $x$ is the position of antenna elements (for example positions 1, 2, 3, etc in Fig. 2). Next we want to determine a factor for the determination of unequal element spacing for ITDU that will be multiplied by the equal element spacing. Let us call this multiplying factor $M(x)$. If the equal element spacing for

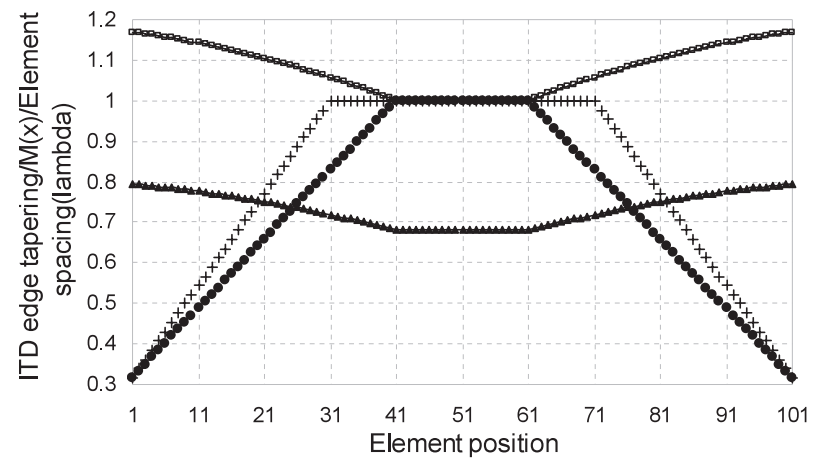

$$
\begin{aligned}
& + \text { Curve } 1 \text { : ITD edge tapering } \\
& \text { - Curve } 2 \text { : ITD for element spacing } A(x) \\
& - \text { Curve } 3 \text { : Multiplying factor for element spacing } M(x) \\
& \rightarrow \text { Curve 4: Element spacing (lambda) }
\end{aligned}
$$

Fig. 2 ITD type edge tapering for 101 phased antenna elements and multiplying factor for unequal spacing of ITDU edge tapered antenna elements. In this figure 30 elements from each side were tapered and 40 elements from each side were of unequal spacing.
ITD is $\mathrm{d}$ then the unequal element spacing for ITDU will be $\mathrm{d} \times M(x)$. And now if we take the sinc function of $A(x)$, the multiplying factor $M(x)$ for the determination of element spacing is to be expressed as Eq. (5):

$$
\begin{aligned}
M(x) & =\operatorname{Sinc}[A(x)] / \min \langle\operatorname{Sinc}[A(x)]\rangle \\
& =\operatorname{Sinc}[A(x)] / 0.8415
\end{aligned}
$$

If the total number of elements is $(2 \mathrm{P}+1)$ then the range of $x$ is from 1 to $2 \mathrm{P}$. Though several other distributions (like Gaussian, cosine, etc.) were tried to determine the unequal spacing it was found that the sinc function of $A(x)$ best fits for the determination of unequal element spacing in order to reduce MSLL and maintain highest BE/BCE. One example of ITD edge tapering, ITD for element spacing, multiplying factor $M(x)$ and element spacing for ITDU of 101 phased array antenna elements is shown in Fig. 2. The steps for the determination of edge tapering and unequal element spacing for ITDU of 1D array are described here:

Step 1: Select the number of antennas to be tapered for unequal power distributions from each side of the phased array antenna according to Curve 1 of Fig. 2. For example 30 elements are selected from both sides for ITDU edge tapering in this figure. Remaining middle 41 elements will of uniform power distribution.

Step 2: Select another Isosceles Trapezoid for the determination of unequal element spacing of ITDU. Curve $2[A(x)]$ is selected for the determination of unequal element spacing in Fig. 2. In this example 40 elements from each side are selected those will be of unequal spacing. Remaining middle 21 elements will of equal spacing.

Step 3: Take the sinc function of curve $2[A(x)]$ to obtain curve $3[M(x)]$ by using Eq. (5).

Step 4: Determine the value of equal element spacing. It was discussed before in Sect. 1 that it is possible to use the antenna spacing between $0.58-0.8 \lambda$ for SPS system. The value of equal element spacing should be chosen in such a way that the value of maximum element spacing remains within this range. The value of equal element spacing is taken $0.68 \lambda$ in this example.

Step 5: Multiply the value of equal element spacing with $M(x)$ to obtain the values of unequal element spacing (curve $4)$. The values of curve 4 are a combination of equal and unequal element spacing. The middle 21 elements of curve 4 are of equal element spacing of $0.68 \lambda$ and the remaining elements are of unequal spacing and the values are symmetric. This is one merit of ITDU over random array/thinned array. Because the values of element spacing in ITDU can be calculated easily from Eq. (5), but the element spacing in random/thinned array is difficult to determine. The fabrication process of ITDU will also be easier than that of random/thinned array. The purpose of taking the difference in number of elements to be tapered and number of elements of unequal spacing is the improvement of beam forming and will be discussed in details later in Sect. 4.

In the following sections the $\mathrm{BE}$ is calculated instead of $\mathrm{BCE}$. If $\mathrm{BE}$ is highest then $\mathrm{BCE}$ will also be highest when 
the size of the rectenna is equal to or greater than the size of the main beam on rectenna. It is also possible to consider antenna "unit" in the following sections. But the number of elements for ITDU and that for other distributions are different. That's why a comparative study is done by considering only elements. The reason of taking the different elements is to keep the same phased array antenna length for ITDU and for other kinds of distributions. Phased array antenna length of 109 elements of equal element spacing of $0.68 \lambda$ (for uniform, Gaussian and ITD edge tapering) is same as the phased array antenna length of 101 elements of ITDU of Fig. 2.

\section{Comparative Study of Different Amplitude Distri- bution System for Different Tapering Level and Beam Steering Angle}

Radiation patterns, MSLL and BE for different tapering levels, inter-element spacing, beam steering angles and for Uniform, Gaussian, ITD and ITDU power distribution systems were compared and summarized in Table 1 . The normalized radiation patterns for case 1 of Table 1 are shown in Fig. 3. Case 1 is for Uniform power distribution (109 elements of spacing $0.68 \lambda),-10 \mathrm{~dB}$ Gaussian edge tapering (109 elements of spacing $0.68 \lambda),-10 \mathrm{~dB}$ ITD (109 elements of spacing $0.68 \lambda),-10 \mathrm{~dB}$ ITDU ((101 elements of spacing ranging from $0.68 \lambda$ to $0.795 \lambda$ ) and $0^{\circ}$ beam steering angle from array broad side. Similarly case 5 of Table 1 is same as case 1 but the beam steering angle is $10^{\circ}$ in this case and the element spacing range is from $0.6 \lambda$ to $0.7 \lambda$ for ITDU. The uniform element spacing for all other distributions in case 5 is $0.6 \lambda$. The reason of reducing the element spacing range for $10^{\circ}$ beam steering angle is to avoid higher SLL in ITDU. This element spacing range is still acceptable for SPS from the view point of maximum beam steering angle, grating lobes and mutual coupling effect.

The power at the center of the transmitter can be made lower in ITD/ITDU than that of Gaussian distribution which is another merit of ITD/ITDU over Gaussian power distribution. One example of power distributions of total 109 watts power for uniform, $-10 \mathrm{~dB}$ ITD, -10 ITDU and $-10 \mathrm{~dB}$ Gaussian edge tapering (case 1) is shown in Fig. 4. The number of antennas in Uniform, Gaussian and ITD edge tapering is 109 and that in ITDU is 101 . The power distribution at the center of the transmitter is the highest in Gaussian distribution which is a demerit because of thermal problem and other technical problems. Element spacing for ITD, Gaussian and uniform distribution was equal. The power distribution at the center of ITDU is higher than that of ITD edge tapering, because the number of antennas is less in ITDU than that in ITD. The power distribution in ITDU can be made identical to that of ITD by considering 109 an-

Table 1 BE and MSLL for different edge tapering systems with different antenna elements, tapering levels, beam steering angles and inter-element spacing.

\begin{tabular}{|c|c|c|c|c|c|c|c|c|c|}
\hline 一 & $N$ & $m$ & $\nabla$ & $n$ & 6 & $r$ & $\infty$ & $a$ & $\stackrel{0}{ }$ \\
\hline 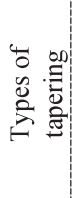 & 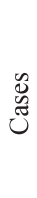 & 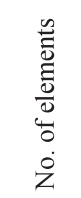 & $\frac{\infty}{0} \overbrace{0}^{\frac{0}{0}}$ & 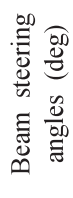 & 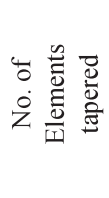 & 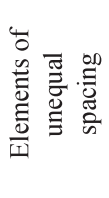 & 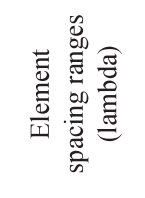 & $\underbrace{2}_{\frac{1}{\infty}}$ & $\begin{array}{l}\underset{\theta}{\theta} \\
= \\
\sum_{\Sigma}^{n}\end{array}$ \\
\hline \multirow{2}{*}{$\stackrel{\Xi}{\varrho}$} & 1 & \multirow[b]{2}{*}{109} & \multirow[b]{2}{*}{0} & 0 & \multirow[b]{2}{*}{0} & \multirow[b]{2}{*}{0} & 0.68 & 89.54 & -13 \\
\hline & 5 & & & 10 & & & 0.6 & 89.8 & -13 \\
\hline \multirow{3}{*}{ 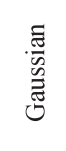 } & 1 & 109 & -10 & \multirow[t]{2}{*}{0} & \multirow{3}{*}{ all } & \multirow{3}{*}{0} & \multirow{2}{*}{0.68} & 98.33 & -22.46 \\
\hline & 2 & 53909 & -10 & & & & & 99.96 & -22.5 \\
\hline & 5 & 109 & -10 & 10 & & & 0.60 & 98.6 & -22 \\
\hline \multirow{5}{*}{$\underline{E}$} & 1 & \multirow{2}{*}{109} & -10 & \multirow{4}{*}{0} & \multirow{2}{*}{33} & \multirow{5}{*}{0} & \multirow{4}{*}{0.68} & 98.30 & -18.7 \\
\hline & 3 & & -20 & & & & & 98.76 & -18.8 \\
\hline & 2 & \multirow{2}{*}{53909} & -10 & & \multirow{2}{*}{16172} & & & 98.31 & -18 \\
\hline & 4 & & -20 & & & & & 98.84 & -18.8 \\
\hline & 5 & 109 & & 10 & 33 & & 0.60 & 98.2 & -19 \\
\hline \multirow{5}{*}{ 忌 } & 1 & \multirow{2}{*}{101} & -10 & \multirow{4}{*}{0} & \multirow{2}{*}{30} & 40 & $0.68 \sim 0.795$ & 98.97 & -26 \\
\hline & 3 & & -20 & & & 37 & $0.68 \sim 0807$ & 99.69 & -32 \\
\hline & 2 & 50001 & -10 & & 1500 & 20000 & $0.68 \sim 0.795$ & 99.999 & -26 \\
\hline & 4 & & -20 & & & 18500 & $0.68 \sim 0807$ & 99.84 & -32 \\
\hline & 5 & 101 & -10 & 10 & 30 & 40 & $0.6 \sim 0.7$ & 99.06 & -26 \\
\hline
\end{tabular}




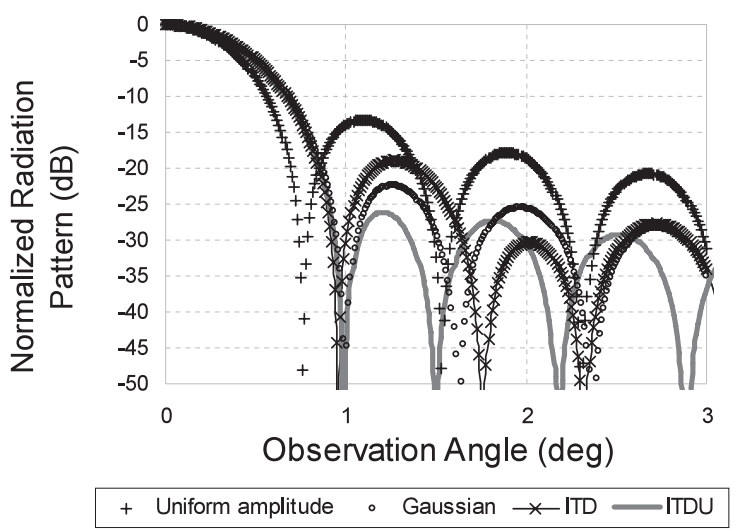

Fig. 3 Normalized radiation patterns for phased array antenna of uniform amplitude, $-10 \mathrm{~dB}$ Gaussian tapering, $-10 \mathrm{~dB}$ ITD and $-10 \mathrm{~dB}$ ITDU. 33 elements from each side were tapered in ITD. 30 elements from each side were tapered in ITDU and 40 elements from each side were of unequal spacing in ITDU.

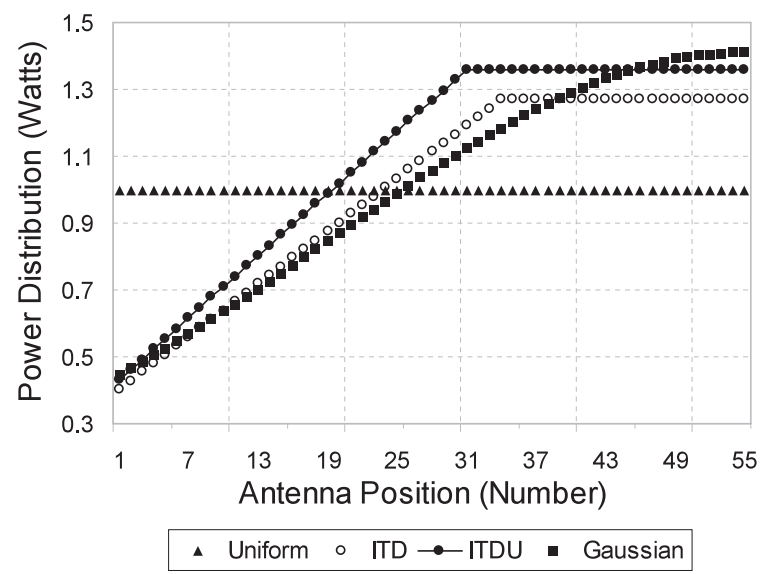

Fig. 4 Uniform amplitude distribution, $-10 \mathrm{~dB}$ ITD (33 elements tapered), $-10 \mathrm{~dB}$ ITDU ( 30 elements tapered and 40 elements are of unequal spacing) and $-10 \mathrm{~dB}$ Gaussian power distribution for total 109 watts power in each case (The number of elements in ITDU is 101 and the number of elements in each of the other cases is 109). Power distributions for half of the arrays are shown in the figure. Power distributions for rest half of the arrays are symmetrical.

tennas in ITDU or by decreasing the number of antennas tapered from both side. But if the number of antennas tapered is decreased then there will be a little increase of MSLL. Radiation pattern for $10^{\circ}$ beam steering angle of ITDU of case 5 is shown in Fig. 5. The MSLL and BE were also measured for 101 phased array antennas of ITDU $(-10 \mathrm{~dB})$ by varying the number of elements tapered and number of elements of unequal spacing from both sides and is summarized in Table 2. It was found that the MSLL was lowest $(-26 \mathrm{~dB})$ when 30 elements were tapered and 40 elements were of unequal spacing from both sides. BE was also the highest $(98.97 \%)$ in this case. Though the MSLL was even lower $(-24.5 \mathrm{~dB})$ in ITDU than that of Gaussian distribution $(-23 \mathrm{~dB})$ when the number of elements tapered was same as the number of elements of unequal spacing in ITDU (30 elements in this case as shown in Table 2). For large number

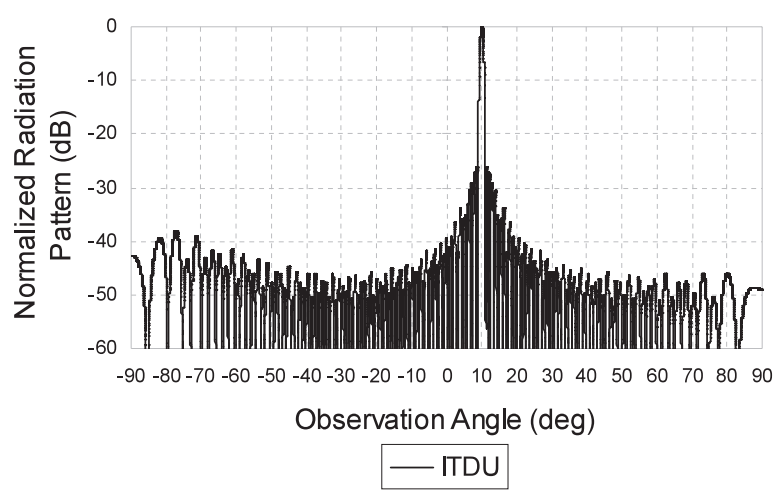

Fig. 5 Normalized radiation pattern for 101 phased array antenna of $-10 \mathrm{~dB}$ ITDU and observation range of $\pm 90 \mathrm{deg}$ for 10 degree beam pointing angle. 30 elements from each side were tapered and 40 elements from each side were of unequal spacing.

Table 2 Different values of MSLL and BE for different number of elements tapered and number of unequal element spacing for 101 phased array antennas and $-10 \mathrm{~dB}$ ITDU tapering.

\begin{tabular}{|c|c|c|c|c|}
\hline $\begin{array}{c}\text { No. of elements tapered from } \\
\text { both side }\end{array}$ & 30 & 30 & 40 & 40 \\
\hline $\begin{array}{c}\text { No. of elements of unequal } \\
\text { spacing from both side }\end{array}$ & 30 & 40 & 30 & 40 \\
\hline MSLL(dB) & -24.5 & -26 & -23 & -22 \\
\hline BE (\%) & 98.70 & 98.97 & 98.22 & 98.50 \\
\hline
\end{tabular}

of antenna elements (case $2 \& 4$ of Table 1 ) and for higher tapering level (case $3 \& 4$ ) ITDU also performed better and can be verified from Table 1 . The observation range was $\pm 0.01^{\circ}$ for case 2 and case 4 . The BE was higher in case 3 $(-20 \mathrm{~dB})$ of ITDU than that of case $1(-10 \mathrm{~dB})$ of ITDU. On the other hand $\mathrm{BE}$ was higher in case $2(-10 \mathrm{~dB})$ of ITDU than that of case $4(-20 \mathrm{~dB})$ of ITDU. The reason of the difference of this BE tendency is that the observation and calculation range for $\mathrm{BE}$ was $\pm 0.01^{\circ}$ (that has been mentioned before) for case 2 and case 4 instead of observation range of $\pm 90^{\circ}$. The observation range and calculation range for $\mathrm{BE}$ for case 1 and case 3 was $\pm 90^{\circ}$.

The BE becomes different for different observation ranges. It was not possible to simulate the radiation patterns for higher number of elements (of case 2 and 4) with higher observation range due to memory problem of the computer.

In case $1-2(-10 \mathrm{~dB}$ tapering $)$ and in case $3-4(-20 \mathrm{~dB}$ tapering) of ITDU the number of elements of unequal spacing were different (column 7 of Table 1). The main reason of this difference is that when 40 elements were chosen from the both side for unequal spacing of $-10 \mathrm{~dB}$ ITDU (case 1) the best MSLL and BE performance was achieved that has already been explained and shown in Table 2 . But for $-20 \mathrm{~dB}$ ITDU (case 3 ) the best MSLL performance was attained when 37 elements were chosen for unequal element spacing. The same reason applies for case 2 and case 4 of ITDU.

From Tables 1, 2 and from Figs. 2-5 it can be seen that the ITDU which is a new concept shows better MSLL/BE performance than any other kinds of distributions, even for 
higher beam steering angle and higher level of tapering. The determination of unequal spacing for ITDU which is within the range of real SPS application is also easier unlike the determination of element spacing for random/thinned array. For example the MSLL for $-10 \mathrm{~dB}$ ITDU and $10^{\circ}$ beam steering angle is $-26 \mathrm{~dB}$ (case 5) and BE for the same situation is $99.06 \%$ and those for Gaussian and ITD of the same case are $-22 \mathrm{~dB} / 98.6 \%$ and $-19 \mathrm{~dB} / 98.2 \%$ respectively.

\section{Experiment with ITDU}

A laboratory experiment on ITDU was conducted by using the SPORTS (Solar POwer Radio Transmission System) $5.8 \mathrm{GHz}$ "beam forming subsystem" in the Microwave Energy Transmission LABoratory of the Kyoto University. A detailed discussion on SPORTS 5.8 is done in [6]. 11 phased array antenna elements with unequal spacing, as shown in Fig. 6, were used during the experiment.

Simulated and measured amplitude distribution of the antenna elements as well as simulated inter-element spacing and inter-element spacing during the experiment are shown respectively in Table 3 and Table 4 . Figure 7 shows the radiation patterns for ITDU and for three different situations of simulation, measurement and simulation with some real errors and the simulated radiation pattern for ITD. From Table 3 and Table 4 it can be seen that there were some dissimilarities between simulation and measurement of amplitude distributions and element spacing. Moreover the attenuators used for the experiment had some phase errors. The assumed amplitude distributions and phase errors those were used during the simulated radiation pattern with errors are shown respectively in rows 3 and 4 of Table 3 . There is a difference between measured and simulated (with errors) radiation patterns after the experiment because it was not possible to find out the exact errors during experiments. There might have been some errors due to reflection from other parts. The purpose of showing the simulated radiation pattern (with errors) is that when these kinds of errors exist in real situation the radiation pattern changes abruptly with increased SLL which is not desirable. The simulated MSLL was about $-28 \mathrm{~dB}$ (2nd SLL in this case) within the observation range of $\pm 30^{\circ}$ and measured MSLL was about $-25 \mathrm{~dB}$ (less than those of $-10 \mathrm{~dB}$ ITD and $-10 \mathrm{~dB}$ Gaussian edge tapering). The MSLL for $-10 \mathrm{~dB}$ Gaussian edge tapering is about $-23 \mathrm{~dB}$ and that of $-10 \mathrm{~dB}$ ITD is about $-20 \mathrm{~dB}$ (Fig. 7). The simulated first SLL (about $-31 \mathrm{~dB}$ ) also agree well with measured first SLL (about $-29 \mathrm{~dB}$ ) in ITDU and can be seen from Fig. 7. Therefore from this experimental and simulated data it can be confirmed that ITDU is the best when it is compared with ITD and Gaussian edge tapering.

\section{ITDU with 2D Array}

The radiation patterns for $-10 \mathrm{~dB}$ and $-20 \mathrm{~dB}$ ITDU with $101 \times 1012 \mathrm{D}$ phased array antennas were studied. The radiation patterns for $-10 \mathrm{~dB}$ ITD and $-10 \mathrm{~dB}$ Gaussian distribution $(109 \times 109$ elements) were also studied. Figure 8

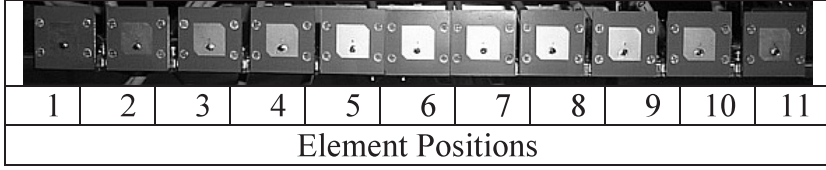

Fig. 6 Phased array antenna elements of SPORTS 5.8 with unequal element spacing for experimentation on ITDU.

Table 3 Simulated and measured amplitude distributions and simulated phase errors of 11 phased array antenna elements and for $-10 \mathrm{~dB}$ ITDU.

\begin{tabular}{|c|c|c|c|c|c|c|c|c|c|c|c|}
\hline $\begin{array}{c}\text { Element } \\
\text { Number } \Rightarrow\end{array}$ & 1 & 2 & 3 & 4 & 5 & 6 & 7 & 8 & 9 & 10 & 11 \\
\hline $\begin{array}{l}\text { Simulated } \\
\text { Amplitude } \\
\text { Distribution }\end{array}$ & $\frac{0}{1}$ & $\begin{array}{l}8 \\
\text { in }\end{array}$ & $\stackrel{\imath}{i}$ & 0 & 0 & 0 & 0 & 0 & $\stackrel{\sim}{\sim}$ & $\begin{array}{l}8 \\
\text { in }\end{array}$ & $\because$ \\
\hline $\begin{array}{l}\text { Measured } \\
\text { Amplitude } \\
\text { Distribution }\end{array}$ & $\begin{array}{l}\frac{9}{1} \\
\stackrel{1}{1}\end{array}$ & 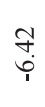 & $\begin{array}{l}6 \\
\dot{5}\end{array}$ & 0 & 0 & 0 & 0 & 0 & & ? & $\stackrel{4}{\stackrel{4}{0}}$ \\
\hline $\begin{array}{l}\text { Phase Error } \\
\text { Considered } \\
\text { during the } \\
\text { Simulation of } \\
\text { Error (deg) }\end{array}$ & $\stackrel{0}{1}$ & $\infty$ & $\simeq$ & 0 & 0 & 0 & 0 & 10 & in & $r$ & $\approx$ \\
\hline
\end{tabular}

Table 4 Simulated and experimental inter-element spacing $(\mathrm{cm})$ of 11 phased array antenna elements and for $-10 \mathrm{~dB}$ ITDU

\begin{tabular}{|c|c|c|c|c|c|c|c|c|c|c|}
\hline Element Numbers $\Rightarrow$ & $I$ & $\stackrel{m}{i}$ & $\stackrel{+}{+}$ & $\stackrel{n}{+}$ & $\begin{array}{l}0 \\
1 \\
1\end{array}$ & 6 & $\stackrel{\infty}{i}$ & $\dot{\infty}$ & $\frac{\circ}{a}$ & $\bar{c}$ \\
\hline $\begin{array}{l}\text { Simulated inter-element } \\
\text { spacing }(\mathrm{cm})\end{array}$ & $\begin{array}{l}m \\
\omega \\
m\end{array}$ & m & $\stackrel{?}{r}$ & $\stackrel{m}{m}$ & $\stackrel{n}{0}$ & $\stackrel{n}{n}$ & $m$ & $\stackrel{m}{m}$ & 吾 & ro \\
\hline $\begin{array}{c}\text { Experimental inter-element } \\
\text { spacing }(\mathrm{cm})\end{array}$ & $\dot{m}$ & $\stackrel{n}{m}$ & $\stackrel{+}{m}$ & $\stackrel{m}{m}$ & $\stackrel{\circ}{\dot{r}}$ & $\stackrel{0}{\ddot{r}}$ & $\stackrel{m}{m}$ & $\ddot{r}$ & $\ddot{n}$ & $\ddot{r}$ \\
\hline
\end{tabular}

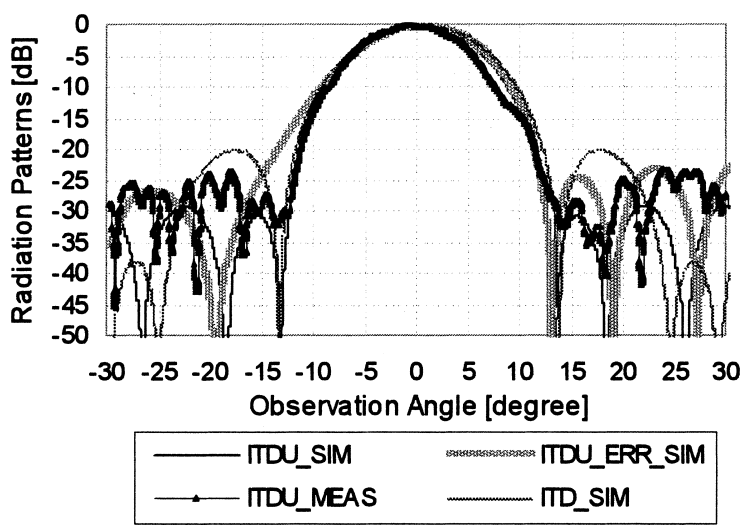

Fig. 7 Simulated, measured and simulated (with error) radiation patterns of ITDU and simulated radiation pattern for ITD with 11 phased array antenna elements.

shows the projected radiation patterns of $-10 \mathrm{~dB}$ ITDU in $\mathrm{XY}, \mathrm{YZ}$ and ZX planes. The MSLL for ITDU, Gaussian and ITD were $-26 \mathrm{~dB},-22 \mathrm{~dB}$ and $-18.75 \mathrm{~dB}$ respectively and BE were $99.82 \%, 99.34 \%$ and $98.82 \%$ respectively for observation angle of $\pm 30^{\circ}$. It can be concluded from this simulation and the 1D simulation case of the same situation that 


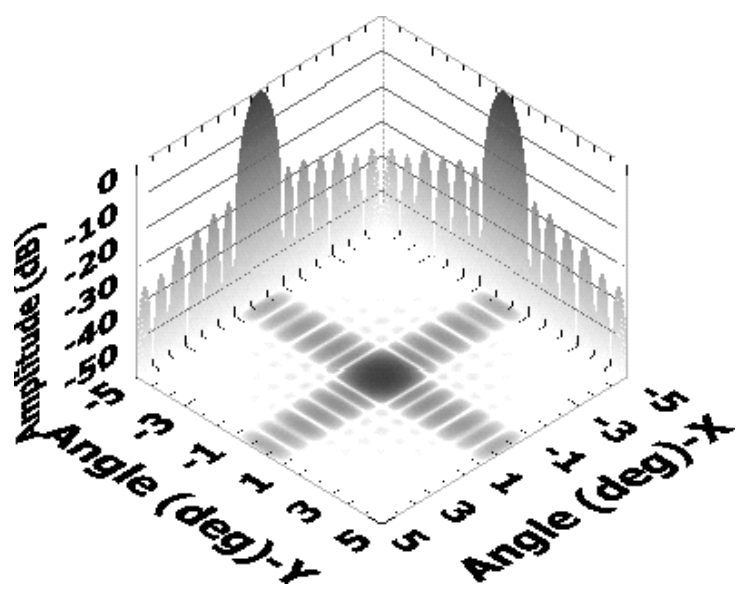

Fig. 8 Three dimensional projected beam pattern for $-10 \mathrm{~dB}$ ITDU of two dimensional arrays in the XY, XZ and YZ planes. Projected pattern in the $\mathrm{XY}$ plane is the contour plot of the radiation pattern.

Table 5 Comparative study of different kinds of amplitude distribution system for two dimensional arrays.

\begin{tabular}{|c|c|c|c|}
\hline $\begin{array}{c}\text { Type of } \\
\text { Tapering }\end{array}$ & $\begin{array}{c}\text { Tapering Level } \\
(\mathrm{dB})\end{array}$ & $\mathrm{MSLL}(\mathrm{dB})$ & $\mathrm{BE}(\%)$ \\
\hline ITDU & -10 & -26 & 99.82 \\
\hline Gaussian & -10 & -22 & 99.34 \\
\hline ITD & -10 & -18.75 & 98.82 \\
\hline
\end{tabular}

the BE for ITDU would be the highest with $-26 \mathrm{~dB}$ MSLL. 30 elements were tapered in ITDU from both sides of the $\mathrm{X}$ and $\mathrm{Y}$ dimensions of the array and 40 elements from both sides of the $\mathrm{X}$ and $\mathrm{Y}$ dimensions were of unequal spacing ranging from $0.68 \lambda$ to $0.795 \lambda$. 33 elements from both sides of $\mathrm{X}$ and $\mathrm{Y}$ dimensions were tapered in ITD. The simulation results are summarized in Table 5. Well performance was also achieved in $-20 \mathrm{~dB}$ ITDU tapering. It is important to mention here that the same rectenna width was considered for different edge tapering system in [6] during the calculation of BCE. But the main beam width is considered, which may be different for different edge tapering/amplitude distribution system, during the calculation of $\mathrm{BE}$.

\section{Conclusions}

Different kinds of amplitude distributions for SPS phased array transmitting antennas are compared and discussed. Edge tapering is needed for higher Beam Efficiency (BE) in Microwave Power Transmission (MPT) but it has thermal and technical complexities. Statistically thinned array antenna can reduce some SLL but there is a possibility of increasing other SLL which can cause reduced BE. Moreover it is difficult to determine the element spacing of thinned/random array. Therefore an optimization is needed between full edge tapering and uniform amplitude distribution. An optimization between full edge tapering and uniform amplitude distribution with equal element spacing is sought by incorporating Isosceles Trapezoidal Distribution (ITD) edge tapering and was discussed in [6]. With ITD it is possible to maintain higher $\mathrm{BCE}$ but higher number of antennas is needed to achieve lower MSLL. Though ITD is still better than Gaussian distribution when BCE, power distribution and other SLL are compared.

Another optimization between ITD and ITD with Unequal spacing (ITDU) to achieve lower MSLL and higher $\mathrm{BE}$ is presented in this paper. It is possible to maintain highest BE and lowest MSLL by incorporating ITDU edge tapering of phased array antenna in MPT system. ITDU is a new concept and it was shown in Tables 1, 2 and Figs. 2-5 that the MSLL/BE performance of ITDU is better than those of ITD and Gaussian distributions, even for higher beam steering angle and higher tapering level. The element spacing for ITDU is very easy to determine and within the range of real SPS application. But the determination of element spacing for thinned/random array is difficult. In case 5 of Table 1 it is shown that the MSLL for $-10 \mathrm{~dB}$ ITDU edge tapering and for $10^{\circ}$ beam steering angle is $-26 \mathrm{~dB}$ and $\mathrm{BE}$ for the same situation is $99.06 \%$. MSLL for ITD and Gaussian edge tapering of case 5 are $-19 \mathrm{~dB}$ and $-22 \mathrm{~dB}$ respectively. BE for ITD and Gaussian are $98.2 \%$ and $98.6 \%$ respectively for case 5 . The performance of ITDU edge tapering for two dimensional case is also same as that of one dimensional case. The power distribution at the centre of the phased array antenna for ITDU case can be made lower than that of Gaussian distribution which is another benefit of ITDU over Gaussian amplitude distribution. Maximum power will be transmitted through the main beam and less power will be in the SLL in case of ITDU even when the same power will be sent for the Gaussian, ITD and ITDU edge tapering. Therefore the exposure level to humans and all other living animals/things outside the rectenna area is less due to ITDU when it is compared to that of Gaussian distribution or ITD. An experimental result of ITDU is also presented which confirmed that better performance of ITDU over ITD and Gaussian edge tapering is possible. Therefore the best form of power distribution for SPS phased array transmitting antenna can be considered to be the ITDU. Further study/experiment on ITDU is needed to test the applicability of the distribution in SPS context.

\section{Acknowledgement}

The authors are grateful to the following people for their cooperation with the laboratory experiment on ITDU: Mr. Miyagawa Tetsuya, Mr. Ohata Yoshiyuki, Mr. Takahiro Hirano, Mr. Shigeaki Kawai and Mr. Fumito Takahashi.

\section{References}

[1] H. Matsumoto, "Research on solar power satellites and microwave power transmission in Japan," IEEE Microw. Mag., vol.3, no.4, pp.36-45, 2002.

[2] D.R. Criswell, "Solar power via the moon," The Industrial Physicist, American Institute of Physics, vol.8, no.2, pp.12-15, April/May 2002.

[3] S. Rahman, "Green power: What is it and where can we find it?" IEEE Power \& Energy magazine, vol.1, no.1, pp.30-37, Jan./Feb. 2003. 
[4] P.E. Glaser, "Power from the sun; its future," Science, vol.162, pp.857-861, Nov. 1968.

[5] W.L. Stutzman and G.A. Thiele, Antenna Theory and Design, 2nd ed., p.296, John Wiley \& Sons, 1998.

[6] A.K.M. Baki, N. Shinohara, H. Matsumoto, K. Hashimoto, and T. Mitani, "Study of isosceles trapezoidal edge tapered phased array antenna for solar power station/satellite," IEICE Trans. Commun., vol.E90-B, no.4, pp.968-977, April 2007.

[7] T. Uno and S. Adachi, "Optimization of aperture illumination for radio wave power transmission," IEEE Trans. Antennas Propag. vol.AP-32, no.6, pp.628-632, June 1984.

[8] C.T. Rodenbeck and K. Chang, "A limitation on the small-scale demonstration of retrodirective microwave power transmission from the solar power satellite," IEEE, Antennas Propag. Mag., vol.47, no.4, pp.67-72, Aug. 2005.

[9] R.M. Dickinson, "Magnetron directional amplifier space power beamer concept design," IECEC, 35th Intersociety Conference, vol.2, pp.1469-1479, July 2000.

[10] F.E. Little, S.J. Kokel, C.T. Rodenbeck, K. Chang, G.D. Arndt, and P.H. Ngo, "Development of a retrodirective control transmitter for wireless power transmission," Radio Science Bulletin, no.311, pp.38-46, Dec. 2004.

[11] J.O. McSpadden and J.C. Mankins, "Space solar power programs and microwave wireless power transmission technology," IEEE Microw. Mag., vol.3, no.4, pp.46-57, 2002.

[12] S.S. Shaposhnikov, R.B. Vaganov, and N.N. Voitovich, "Antenna amplitude distributions for improved wireless power transmission efficiency," IEEE AFRICON. 6th, vol.2, pp.559-562, Oct. 2002

[13] R.B. Vaganov, "Maximum power transmission between two apertures with the help of a wave beam," J. Communication Technology and Electronics, vol.42, no.4, p.430, 1997.

[14] Y. Rahmat-Samii, D. Gies, and J. Robinson, "Particle swarm optimization (PSO): A novel paradigm for antenna designs," Radio Science Bulletin, no.305, pp.14-22, Sept. 2003.

[15] Fung.I. Tseng, "Design of array and line-source antennas for Taylor patterns with a null," IEEE Trans. Antennas Propag., vol.AP-27, no.4, pp.474-479, July 1979.

[16] A. Safaai-Jazi, "A new formulation of the design of Chebyshev arrays," IEEE Trans. Antennas Propag., vol.42, no.3, pp.439-443, 1994.

[17] V.R. Garmash, B.Z. Katsenelenbaum, S.S. Shaposhnikov, V.N. Tioulpakov, and R.B. Vaganov, "Some pecularities of the wave beams in wireless power transmission," IEEE Aerosp. Electron. Syst. Mag., vol.13, no.10, pp.39-41, Oct. 1998.

[18] V.N. Garmash and S.S. Shaposhnikov, "Matrix method synthesis of transmitting antenna for wireless power transmission," IEEE Trans. Aerosp. Electron. Syst., vol.36, no.4, pp.1142-1148, Oct. 2000.

[19] M.I. Skolnik, J.W. Sherman, III, and F.C. Ogg, Jr., "Statistically designed density-tapered arrays," IEEE Trans. Antennas Propag., vol.12, no.4, pp.408-417, July 1964

[20] W.C. Brown and E. Eugene Eves, "Beamed microwave power transmission and its application to space," IEEE Trans. Microw. Theory Tech., vol.40, no.6, pp.1239-1250, June 1992.

[21] G. Goubau and F. Schwering, "On the guided propagation of electromagnetic wave beams," IRE Trans. Antennas Propag., vol.AP-9, pp.248-256, 1961.

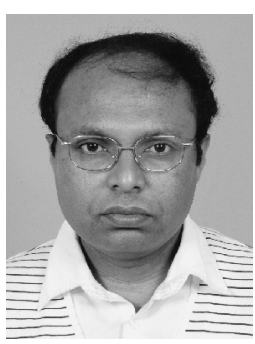

A.K.M. Baki received B.Sc. in Electrical and Electronic Engineering degree from Bangladesh University of Engineering and Technology (BUET), Bangladesh in 1992 and his M.Sc. degree from the University of Bolton (former Bolton Institute of Higher Education), UK in 2003 having followed a postgraduate program in Electronic System and Engineering Management at the South-Westphalia University of Applied Science, Germany. He received Ph.D. degree in Electrical Engineering from Kyoto University, Kyoto, Japan in 2007. From 2005 to 2006 he was a research assistant in the Research Institute for Sustainable Humanosphere (RISH), Kyoto University by recognizing the Radio Science Center for Space and Atmosphere (RASC). From 2004 to 2005 he was a research assistant in the Radio Science Center for Space and Atmosphere (RASC), Kyoto University. From 2003 to 2004 he was a research student in Radio Science Center for Space and Atmosphere (RASC), Kyoto University. From 2007, he has been an Assistant Professor in the School of Engineering and Computer Science (SECS) of the Independent University, Bangladesh. From 2003 to 2007 he was a Sub-Divisional Engineer in Bangladesh Power Development Board (BPDB). From 1994 to 2003 he was an Assistant Engineer in BPDB. He is the former recipient of scholarship from German Academic Exchange Service (DAAD - DEUTSCHER AKADEMISCHER AUSTAUSCHDIENST) for the study of his M.Sc. degree. He received Japan Government (Monbukagakusho: MEXT) scholarship for the study of his Ph.D. degree. He is a member of the IEEE.

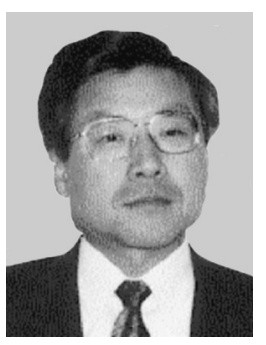

Kozo Hashimoto received the B.E., M.E. and $\mathrm{Ph} . \mathrm{D}$. (Eng.) degrees in electrical engineering from Kyoto University, Japan, in 1969, 1971 and 1980, respectively. He was a research associate in the Faculty of Engineering, Kyoto University from 1974 to 1985 . He was an associate professor in the Faculty of Engineering, Tokyo Denki University from 1985 to 1989 , and there he was a professor from 1989 to 1995 . He was a professor in the Radio Atmospheric Science Center, Kyoto University from 1995, and a professor in the Radio Science Center for Space and Atmosphere (RASC), Kyoto University by reorganizing the Radio Atmospheric Science Center from 2000. Since 2004, he has been a professor in Research Institute for Sustainable Humanosphere, Kyoto University by reorganizing the RASC. He has been engaged in research on Solar Power Station/Satellite, Microwave Power Transmission system, wave phenomena in the Earth's magnetosphere plasma, and development of plasma wave analyzer. He received the Tanaka Date Award from Society of Terrestrial Magnetism and Electricity of Japan in 1984. He is a member of American Geophysical Union (AGU), a member of Society of Geomagnetism and Earth, Planetary and Space Sciences (SGEPSS) and a senior member of the IEEE. 


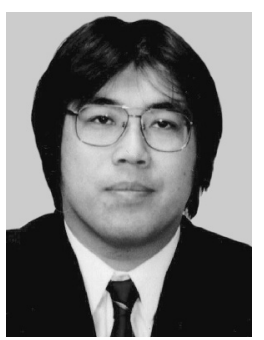

Naoki Shinohara received the B.E. degree in electronic engineering, the M.E. and Ph.D. (Eng.) degrees in electrical engineering from Kyoto University, Japan, in 1991, 1993 and 1998, respectively. He was a research associate in the Radio Atmospheric Science Center, Kyoto University from 1998. He was a research associate of the Radio Science Center for Space and Atmosphere, Kyoto University by recognizing the Radio Atmospheric Science Center from 2000, and there he was an associate professor since 2001. From 2004, he has been an associate professor in Research Institute for Sustainable Humanosphere, Kyoto University by recognizing the Radio Science Center for Space and Atmosphere. He has been engaged in research on Solar Power Station/Satellite and Microwave Power Transmission system. He is a member of the IEEE and IEEJ.

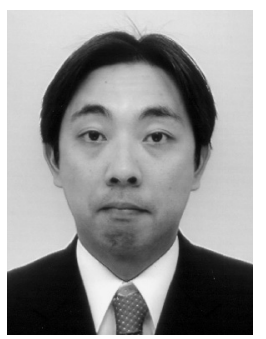

Tomohiko Mitani received the B.E. degree in electrical and electronic engineering, the M.E. degree in informatics, and the Ph.D. degree in electrical engineering from Kyoto University, Kyoto, Japan, in 1999, 2001, and 2006, respectively. He was an Assistant Professor with the Radio Science Center for Space and Atmosphere, Kyoto University, in 2003. He has been an Assistant Professor with the Research Institute for Sustainable Humanosphere, Kyoto University, since 2004. His current research interests include the experimental study on magnetrons, microwave power transmission systems, and applied microwave engineering. He is a member of IEEE.

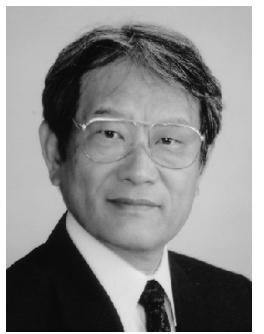

Hiroshi Matsumoto received the B.E. degree in electronic engineering, the M.E. and Ph.D. (Eng.) degrees in electrical engineering from Kyoto University, Japan, in 1965, 1967 and 1973, respectively. He was a professor of the Radio Atmospheric Science Center, Kyoto University from 1977, and there he was the director from 1992 to 1998 . He has been a professor in the Radio Science Center for Space and Atmosphere, Kyoto University by recognizing the Radio Atmospheric Science Center since 2000 and there he was the director from 2002 to 2004. From 2004 to 2005, $\mathrm{He}$ was a professor and director in Research Institute for Sustainable Humanosphere, Kyoto University by recognizing the Radio Science Center for Space and Atmosphere. From 2005, he has been an executive vice president of Kyoto University. He has been an advisory board in Communications Research Laboratory, Japan since 2001. He has been engaged in research on space plasma physics, space radio science and engineering, Solar Power Station/Satellite and Microwave Power Transmission system. He received the Tanaka Date Award from Society of Terrestrial Magnetism and Electricity of Japan in 1975, the NASA Group Achievement Award in 1993 and 1998, the American Geophysical Union (AGU) Fellow Award in 1999, the Shida-Rinzaburo Award in 1999 and the ISAP2000 Paper Award in 2000 and IEEE Fellow Award in 2003. He is an AGU Fellow, a member of Society of Geomagnetism and Earth, Planetary and Space Sciences (SGEPSS) and an IEEE Fellow. He was the president of the International Union of Radio Science (URSI) from 1999 to 2002. 